\title{
Electrochemical Properties of Polyaniline Electrodes Prepared by Chemical Synthesis and Electrodeposition: Revisited with High- Scan-Rate Behaviors
}

\author{
Ji Hyun Namª ${ }^{\mathrm{a}}$, Cho Hyeon $\mathrm{Woo}^{\mathrm{a}}$, Kwang Man Kim ${ }^{\mathrm{b}, \boldsymbol{\dagger}}$, Kwang Sun Ryu ${ }^{\mathrm{c}}$, and Jang Myoun Ko ${ }^{\mathrm{a}, \uparrow}$ \\ ${ }^{a}$ Department of Chemical and Biological Engineering, Hanbat National University, Daejon 305-719, Republic of Korea \\ ${ }^{b}$ Research Team of Power Control Devices, Electronics \& Telecommunications Research Institute (ETRI), Daejon 305-700, \\ Republic of Korea \\ ${ }^{c}$ Department of Chemistry, University of Ulsan, Ulsan 680-749, Republic of Korea
}

\begin{abstract}
:
The polyaniline (PANI) electrodes are prepared by chemical synthesis and electrodeposition methods and their supercapacitive properties are characterized and compared by morphology observation, cyclic voltammetry as a function of scan rate, and impedance spectra analysis. In particular, the supercapacitive properties obtained in the range of higher potential scan rates (e.g., over $200 \mathrm{mV} \mathrm{s}^{-1}$ ) are emphasized to be capable of utilizing adequately the high power capability of supercapacitor. As a result, the PANI electrode by the electrodeposition shows superior specific capacitance (max. $474 \mathrm{~F} \mathrm{~g}^{-1}$ at $10 \mathrm{mV} \mathrm{s}^{-1}$ and about $390 \mathrm{~F} \mathrm{~g}^{-1}$ at $500 \mathrm{mV} \mathrm{s}^{-1}$ ) than those by the chemical synthesis method. This is mainly due to highly porous structure obtained by the electrodeposition to yield higher specific surface area.
\end{abstract}

Keywords: Polyaniline, Chemical synthesis, Electrodeposition, Supercapacitive property

Received May 17, 2012 : Accepted June 15, 2012

\section{Introduction}

Polyaniline (PANI) is one of most commonly used conducting polymers as an electrode of supercapacitors, ${ }^{1-5)}$ due to its high conductivity, stable redox behavior, environmental friendliness, and even good processability. In early stage for supercapaitor application, chemically synthesized PANI electrodes with different routes achieved comparatively low specific capacitances, e.g., 237 or $335 \mathrm{~F} \mathrm{~g}^{-1}$ at $100 \mathrm{mV} \mathrm{s}^{-1}$, 5,6) due to compact granular morphology which is similar to previous reports. ${ }^{7,8)}$ However, the specific capacitance of PANI electrode could be improved by adopting various synthesis techniques. For instance, the

Corresponding authors. Tel.: +82-42-821-1545

E-mail address: jmko@hanbat.ac.kr (J.M. Ko) or kwang@etri.re.kr (K.M. Kim) nanograined PANI prepared by a chemical bath deposition showed a specific capacitance of $503 \mathrm{~F} \mathrm{~g}^{-1}$ at $10 \mathrm{mV} \mathrm{s}^{-1}$. ${ }^{9)}$ The PANI thin film by a microwaveassisted chemical deposition exhibited $753 \mathrm{~F} \mathrm{~g}^{-1}$ at $5 \mathrm{mV} \mathrm{s}^{-1}{ }^{10)}$ The electrochemical deposition of PANI yielding the shapes of nanorod, ${ }^{11)}$ nanowire, ${ }^{12)}$ and nanowire networks ${ }^{13,14)}$ greatly improved the specific capacitance up to $839 \mathrm{~F} \mathrm{~g}^{-1}$ at the low scan rate of $10 \mathrm{mV} \mathrm{s}^{-1}$.

However, such the higher capacitance values were obtained in the range of comparatively low scan rates, e.g., $5-200 \mathrm{mV} \mathrm{s}^{-1}$, as summarized in Fig. 3. This figure is introduced in advance for comparison of the previously reported capacitance results as a function of potential scan rate. The nanostructured PANI electrodes obtained by chemical and electrochemical depositions showed very steeply decreasing pattern of capacitance in the range of low scan rates. Meanwhile, 
the bulk PANI electrodes obtained by chemical synthesis exhibited a monotonously decreasing pattern of capacitance in the range of high scan rates. It is thus curious that the steeply decreasing pattern may be relaxed to a monotonous curve with high capacitance values in the range of high scan rates.

In this study, we simply test the supercapacitive properties in a wide scan rate region, covering both the low and high scan rate regions, for PANI electrodes obtained by a chemical synthesis and an electrodeposition method. The PANI electrode by the chemical synthesis is prepared as two samples with and without adopting carbon black to examine the effect of conductive agent. The readers should bear in mind that this study just compares the supercapacitive properties of bulk PANI electrodes, not nanostructured PANIs, obtained by simple chemical synthesis and electrodeposition technique.

\section{Experimental}

The PANI powder by chemical synthesis was obtained by following procedures: $7 \mathrm{~g}$ of aniline monomer (Junsei) was added to the $300 \mathrm{~mL}$ of $1 \mathrm{M}$ $\mathrm{HClO}_{4}$ solution and sonicated to achieve good dispersion. After sonication, the solution was cooled down to $1^{\circ} \mathrm{C}$. About $200 \mathrm{~mL}$ of $1 \mathrm{M} \mathrm{HClO}_{4}$ solution containing ammonium persulfate oxidant was then added to the monomer solution drop by drop for $1 \mathrm{~h}$ with mechanically stirring at $300 \mathrm{rpm}$. The stirring was continued overnight after the temperature increased to $15^{\circ} \mathrm{C}$. The resultant solid was then filtered, washed, and dried at $40^{\circ} \mathrm{C}$ to yield the PANI powder. The Brunauer-Emmett-Teller (BET) surface area was measured using a Smartsorb-92/93 and then resulted in $82 \mathrm{~m}^{2} \mathrm{~g}^{-1}$. The morphology of the PANI powder was examined using a scanning electron microscope (Jeol, JSM-6300). Two kinds of PANI electrode were prepared from the PANI powder obtained by chemical synthesis: (i) the electrode consisting of PANI powder (90 wt \%) mixed with poly(vinylidene fluoride) (Aldrich) $(10 \mathrm{wt} \%)$ [designated as cPANI (no Super P)], and (ii) the electrode consisting of PANI powder (90 wt\%) mixed with carbon black (Super P, Timcal) (5 wt\%) and poly(vinylidene fluoride) (Aldrich) $(5 \mathrm{wt} \%$ ) [designated as cPANI (Super P)]. The loading density of PANI as an active material in the chemically synthesized PANI electrodes was determined as $1.10 \mathrm{mg} \mathrm{cm}^{-2}$.
The electrodeposition of PANI on a Pt substrate was carried out potentiodynamically using an Autolab instrument (PGstat 100, Eco-Chemie) by subjecting the working electrode to potential cycling between -0.05 and $0.85 \mathrm{~V}$ at a scan rate of $500 \mathrm{mV} \mathrm{s}^{-1}$ in an aqueous electrolyte solution consisting of $0.1 \mathrm{M}$ aniline and $0.2 \mathrm{M} \mathrm{H}_{2} \mathrm{SO}_{4}$ for 150 cycles under $\mathrm{N}_{2}$ atmosphere. An electrochemical cell was assembled in a three-electrode configuration in which the working and counter electrodes were $\operatorname{Pt}\left(1 \times 1 \mathrm{~cm}^{2}\right)$, and the reference electrode was an $\mathrm{Ag} / \mathrm{AgCl}$ (saturated $\mathrm{KCl}$, $0.222 \mathrm{~V}$ vs. SHE). The BET surface area measured was evaluated as $168 \mathrm{~m}^{2} \mathrm{~g}^{-1}$. The loading density of PANI as an active material in the electrodeposited PANI electrode [designated as ePANI] was determined as $0.11 \mathrm{mg} \mathrm{cm}^{-2}$. The morphology of the PANI electrodeposited was also examined using a scanning electron microscope (Jeol, JSM-6300).

The supercapacitive properties of the PANI electrodes were mainly obtained by cyclic voltammetry measurement using an Autolab instrument (PGstat 100, Eco-Chemie). An Ag/AgCl (saturated KCl, 0.222 $\mathrm{V}$ vs. SHE) reference electrode and Pt counter electrode $\left(2 \times 2 \mathrm{~cm}^{2}\right)$ were used to control the potentials of the working electrode between -0.2 and $0.6 \mathrm{~V}$ (vs. $\mathrm{Ag}$ / $\mathrm{AgCl})$ in $1 \mathrm{M} \mathrm{H}_{2} \mathrm{SO}_{4}$ aqueous solution at various scan rates $\left(10-500 \mathrm{mV} \mathrm{s}^{-1}\right)$. Complex impedance measurements were also made for the PANI electrodes using an Autolab instrument equipped with a frequency response analyzer in the frequency range of $10^{-2}$ $10^{4} \mathrm{~Hz}$ with an alternating current perturbation of $5 \mathrm{mV}$.

\section{Results and Discussion}

Fig. 1 shows powder morphologies of PANI prepared by chemical synthesis (cPANI) and electrodeposition (ePANI). The cPANI has slightly elongated particles or particle aggregates with an average diameter and length of about 100 and $400 \mathrm{~nm}(L / D=\sim 4)$, respectively, which are distributed randomly with some distinguishable pores and surface roughness. The morphology of cPANI is very similar to the porous nanorod PANI film obtained by electrochemical deposition, ${ }^{15)}$ but the cPANI in this study do not contain any nanostructure with interconnected nanonetworks. In contrast, the ePANI on Pt substrate has compactly connected particles or particle aggregates (their sizes are below $70 \mathrm{~nm}$ ), which are to some 


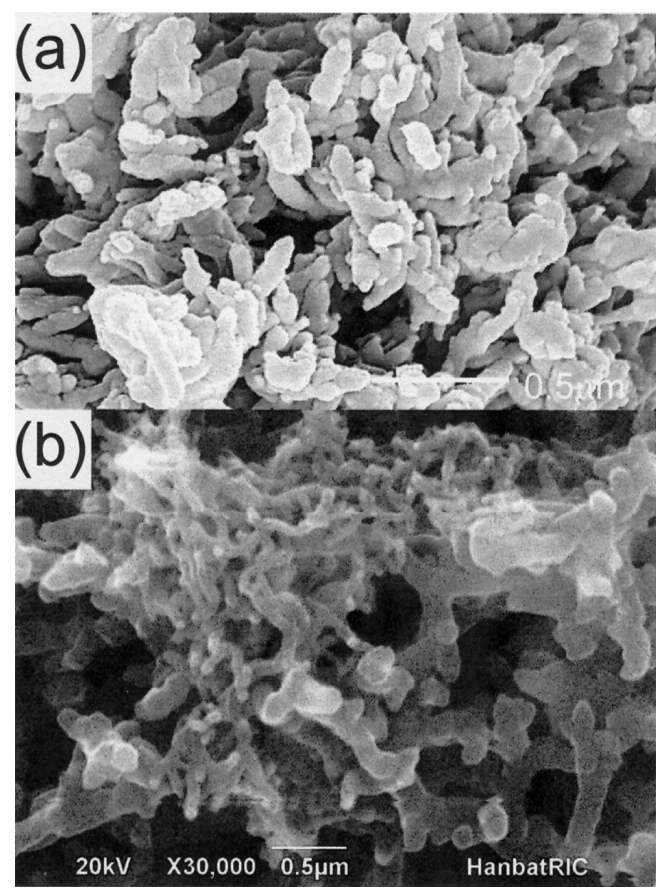

Fig. 1. Scanning electron microscopic images of (a) PANI powder synthesized chemically and (b) PANI electrode electrodeposited by potentialdynamic cyclic voltammetry technique. extent localized with higher specific surface area $\left(168 \mathrm{~m}^{2} \mathrm{~g}^{-1}\right)$ that that of cPANI $\left(82 \mathrm{~m}^{2} \mathrm{~g}^{-1}\right)$. Such the nearly double difference in the specific surface area may be a contributing factor to the capacitive properties of these PANI for supercapacitor electrode application.

Cyclic voltammetry results for the ePANI and cPANI electrodes (with and without Super P) are shown in Fig. 2, obtained in the range of potential scan rate of (10-500 $\left.\mathrm{mV} \mathrm{s}^{-1}\right)$. As shown in Fig. 2(d), current density response of ePANI is higher than that of cPANI, implying more stable supercapacitive characteristics. ${ }^{12)}$ The difference in the redox behaviors between cPANI electrodes (with and without Super P) does not distinguishable, because in this study the powder property (e.g., porosity) seems to suppress efficiently the conductivity enhancing effect by the addition of conductive agent. Also, all the PANI electrodes, irrespective of chemical synthesis and electrodeposition, show that only a broad redox process is observed but shifting the peak potential with increasing the scan rate. The results indicate that the PANI electrode has obvious capacitive performance with a couple of Faradaic peaks relating to the protonation and deprotonation processes. ${ }^{15)}$ The shift of peak
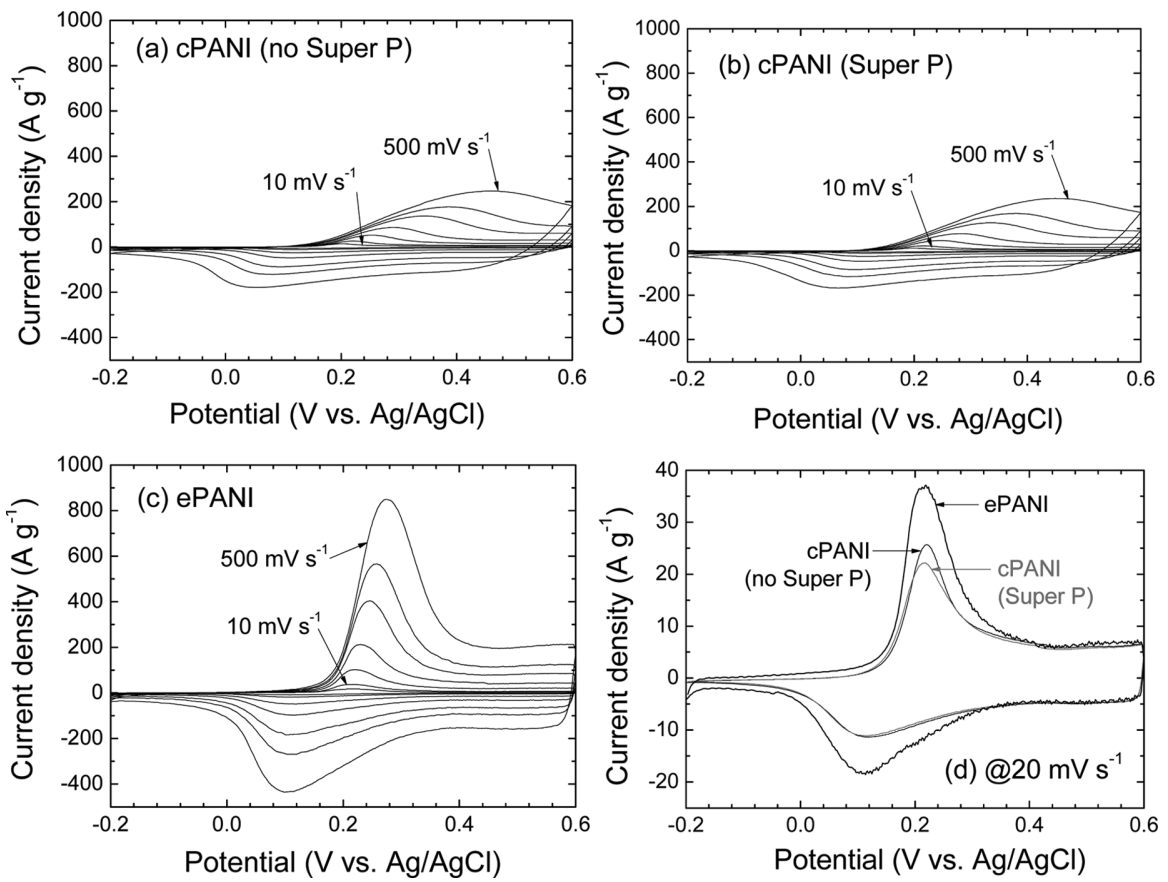

Fig. 2. Cyclic voltammograms of (a) the cPANI (no Super P), (b) the cPANI (Super P), and (c) the ePANI. Cyclic voltammograms of all the PANI electrodes, recorded at $20 \mathrm{mV} \mathrm{s}^{-1}$, are shown in (d). 


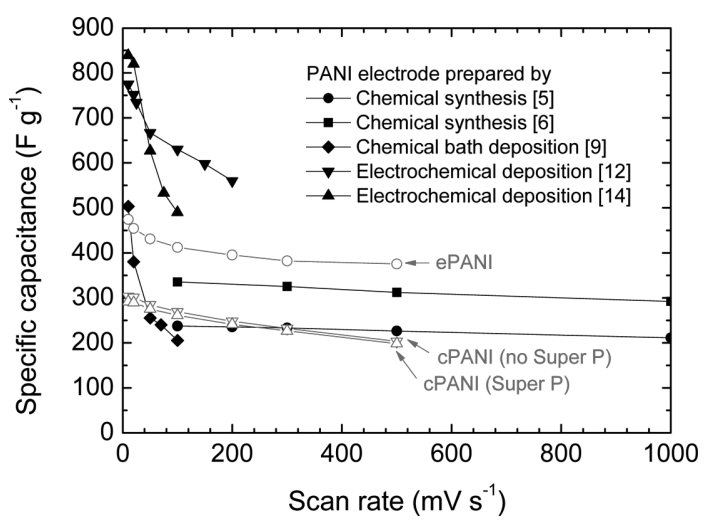

Fig. 3. Specific capacitance evolution as a function of scan rate. Data for cPANI and ePANI only were calculated from the cyclic voltammograms in Fig. 2. All other data were quoted from the previous reports. ${ }^{5,6,9,12,14)}$

potential may be due to growing scan rate where the redox process occurs. It is also interesting to note that the rectangular shape of cyclic voltammograms at higher potential region for the ePANI electrode (see Fig. 2(c)) indicates the presence of pseudocapacitance characteristics, which are distinct from the electrical double layer capacitance. ${ }^{9}$

Specific capacitance values $(C)$ can be calculated from the cyclic voltammogram using an equation of $C=\left(q_{\mathrm{a}}+q_{\mathrm{c}}\right) /(2 m \Delta \mathrm{V})$, where $q_{\mathrm{a}}, q_{\mathrm{c}}, m$, and $\Delta V$ denote anodic and cathodic charges on each scan, mass of the active material, and the potential window, respectively. Fig. 3 shows specific capacitance of PANI electrodes as a function of scan rate, as well as summarized results from the previous reports. $5,6,9,12,14$ ) Comprehensively considering, the supercapacitor should also be viewed at the high-scan-rate region, corresponding to its typical feature of high-rate capability. Some previous reports ${ }^{9,12,14)}$ only concerns at the low-scan rate region that typically exhibits steeply decreasing capacitance with the increase in scan rate. This study extends the scan rate region up to $500 \mathrm{mV} \mathrm{s}^{-1}$ and it is expected that the specific capacitance can saturate its value to some extent over the scan rate. As a conclusion, the ePANI reaches a maximum specific capacitance of $474 \mathrm{~F} \mathrm{~g}^{-1}$ at $10 \mathrm{mV} \mathrm{s}^{-1}$ and about $390 \mathrm{~F} \mathrm{~g}^{-1}$ at $500 \mathrm{mV} \mathrm{s}^{-1}$ (with the capacitance retention ratio of $82.3 \%$ ), which are higher than those of cPANI electrodes. This is probably why the more porous property and thus the higher surface area of ePANI contribute to the capacitance increase, rather than the conductivity enhancement by, e.g., the addi-
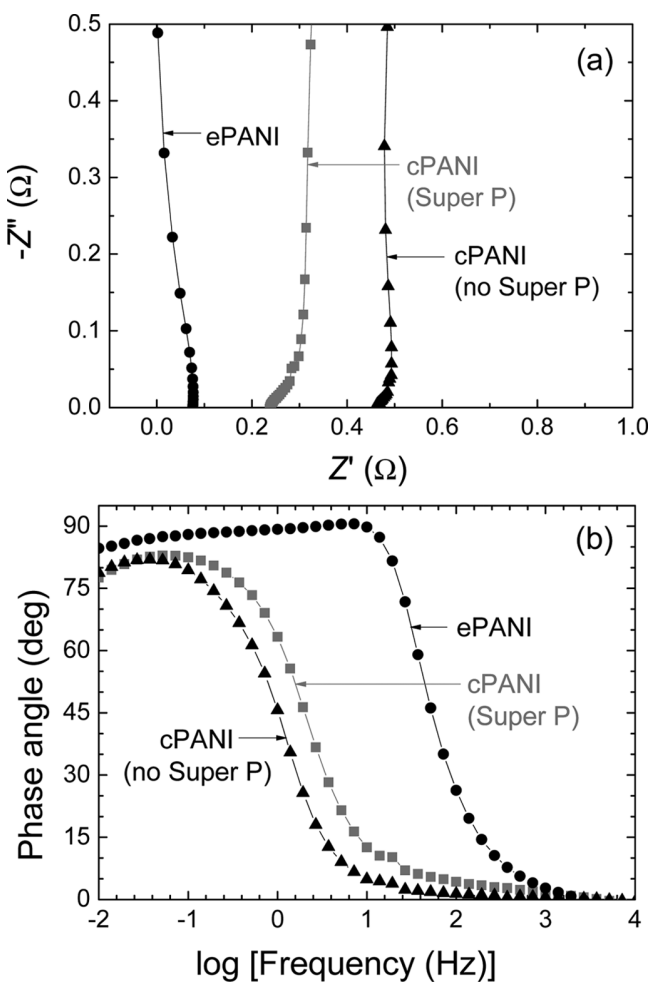

Fig. 4. (a) Nyquist and (b) Bode plots of the cPANI (no Super P), the cPANI (Super P), and the ePANI electrodes.

tion of conductive agent.

The suppression of conductive agent effect can also be evidenced by the Nyquist plot in Fig. 4(a), obtained from complex impedance measurement. As expected, the cPANI electrode with Super P exhibits lower bulk resistance than the cPANI without Super P, due to the conductivity increase to a certain extent. However, the ePANI electrode shows lowest bulk resistance compared to the cPANI electrodes. This can be resulted from the conductivity promoted by the compactly deposited ePANI and the porous property of ePANI surely suppresses the contribution of conductivity increase by the addition of conductive agent to cPANI electrode. Also, Bode plot in Fig. 4(b) indicates response time at the phase angle of $45^{\circ}$. The ePANI electrode gives faster response time than the cPANI electrodes, implying that ionic mobility for charge storage is faster ${ }^{16)}$ in ePANI than cPANI electrodes.

\section{Conclusions}

This study is focused mainly on the high-scan-rate 
supercapacitive behavior of PANI electrodes, prepared by chemical synthesis and electrodeposition. It is aimed to correspond to real utilization of supercapacitor as typical power storage device with high-power input/output. As a result, the electrodeposited PANI electrode with the compactly connected particles or particle aggregates (their sizes are below $70 \mathrm{~nm}$ ) can achieve a saturated specific capacitance of $390 \mathrm{~F} \mathrm{~g}^{-1}$ at $500 \mathrm{mV} \mathrm{s}^{-1}$, which promises an adequate electrode material for long-endured high-power supercapacitor application.

\section{Acknowledgements}

This research was financially supported by the Ministry of Education, Science Technology (MEST) and National Research Foundation of Korea (NRF) through the Human Resource Training Project (201106-04-055) for Regional Innovation in Korea. One of authors (KMK) appreciates partial financial support by the Converging Research Center Program through the Korean Ministry of Education, Science and Technology (2012K001259).

\section{References}

1. K. S. Ryu, K. M. Kim, N.-G. Park, Y. J. Park, and S. H. Chang, J. Power Sources, 103 , 305 (2002).

2. J. M. Ko, R. Y. Song, H.-H. Ju, J. W. Yoon, B. G. Kim, and D. W. Kim, Electrochim. Acta, 50, 873 (2004).

3. K. S. Ryu, S. K. Jeong, J. Joo, and K. M. Kim, J. Phys. Chem. B., 111, 731 (2007).

4. K. S. Ryu and K. M. Kim, J. Power Sources, 165, 420 (2007).

5. J. M. Ko, K. S. Ryu, S. Kim, and K. M. Kim, J. Appl. Electrochem, 39, 1331 (2009).

6. R. Y. Song, J. H. Park, S. R. Sivakkumar, S. H. Kim, J. M. Ko, D.-Y. Park, S. M. Jo, and D. Y. Kim, J. Power Sources, 166, 297 (2007).

7. S. R. Sivakkumar and D.-W. Kim, J. Electrochem. Soc., 154, A134 (2007).

8. K. S. Ryu, X. Wu, Y.-G. Lee, and S. H. Chang, J. Appl. Polym. Sci., 89, 1300 (2003).

9. D. S. Dhawale, A. Vinu, and C. D. Lokhande, Electrochim. Acta, 56, 9482 (2011).

10. P. R. Deshmukh, S. N. Pusawale, V. S. Jamadade, U. M. Patil, and C. D. Lokhande, J. Alloys Comp., 509, 5064 (2011).

11. C. A. Amarnath, J. H. Chang, D. Kim, R. S. Mane, S.-H. Han, and D. Sohn, Mater. Chem. Phys., 113, 14 (2009).

12. V. Gupta and N. Miura, Mater. Lett., 60, 1466 (2006).

13. V. Gupta and N. Miura, Electrochem. Solid-State Lett., 8, A630 (2005).

14. D. S. Dhawale, D. P. Dubal, V. S. Jamadade, R. R. Salunkhe, and C. D. Lokhande, Synth. Metals, 160, 519 (2010).

15. J. Tu, J. Hou, W. Wang, S. Jiao, and H. Zhu, Synth. Metals, 161, 1255 (2011).

16. K. S. Ryu, Y.-G. Lee, K. M. Kim, Y. J. Park, Y.-S. Hong, X. Wu, M. G. Kang, N-G. Park, R. Y. Song, and J. M. Ko, Synth. Metals, 153, 89 (2005). 